\title{
Short Term Efficacy of Entecavir in the Treatment of Decompensated Chronic Hepatitis B Cirrhosis
}

\author{
Weiwei $\mathrm{Fu}$
}

Third People's Hospital of Zhenjiang City, Zhenjiang 212001, Jiangsu Province, China

\begin{abstract}
Objective: To explore the effect of entecavir on patients with decompensated chronic hepatitis B cirrhosis. Methods: From October 2007 to December 2019, 100 patients with decompensated chronic hepatitis B cirrhosis who were treated in our hospital were selected to carry out this study. The clinical data of the patients were analyzed. According to whether entecavir treatment was carried out, 100 patients were divided into two groups, 50 cases in the control group and 50 cases in the observation group. The control group was treated with conventional drugs, and the observation group was treated with entecavir. Liver function indexes, liver fibrosis indexes, HBV-DNA negative conversion rate and incidence of adverse reactions were compared between the two groups. Results: Compared with the control group, the liver function indexes of the observation group were lower, $P<0.05$; Compared with the control group, the observation group was better, $P<0.05$; The negative rate of HBV-DNA in the observation group was lower than that in the control group $(P<0.05)$; There was no difference in the incidence of adverse reactions between the two groups, $P>0.05$. Conclusion: Entecavir can not only improve the liver function, but also enhance the shortterm treatment effect, without increasing adverse reactions, and has high safety, which is worthy of recommendation.
\end{abstract}

Keywords: Decompensated stage of chronic hepatitis B cirrhosis; Entecavir; Treatment effect; Negative rate of HBV-DNA

Publication date: November, 2020

Publication online: 30 November, 2020

"Corresponding author: Weiwei Fu, 32646870@ qq.com

Chronic hepatitis B cirrhosis is a common disease, and its occurrence is mostly caused by the long-term replication of chronic hepatitis B virus, resulting in the destruction of liver cells. Such disease can not be treated in time. When patients enter the decompensated period, it will aggravate the patient's condition and threaten their life safety ${ }^{[1]}$. The decompensated stage belongs to the late stage of patients with decompensated cirrhosis of hepatitis B. At this time, there are many complications, so the mortality rate is high ${ }^{[2]}$. At present, antiviral drugs are often used in the treatment of this disease in order to inhibit the replication of the virus, block the activity of the virus and control the development of the disease. However, practice shows that in the decompensated period of patients with chronic hepatitis B cirrhosis, entecavir can improve the negative conversion rate of HBV-DNA and the treatment effect, and improve the liver function of patients ${ }^{[3]}$. In this study, 100 patients as the background, to explore the effect of entecavir on patients with decompensated chronic hepatitis B cirrhosis

\section{Material and methods}

\subsection{General information}

From October 2007 to December 2019, 100 patients with decompensated chronic hepatitis B cirrhosis who were treated in our hospital were selected to carry out this study. The clinical data of the patients were analyzed. According to whether entecavir treatment was carried out, 100 patients were divided into two groups, 50 cases in the control group and 50 cases in the observation group. In the control group, 
there were 31 males and 19 females with an average age of $(55.45 \pm 10.25)$ years (range, $42-68$ years). The course of disease was 2.5-23.5 years, with an average of (13.12 \pm 4.08$)$ years; Observation group: there were 30 males and 20 females with an average age of (55.11 \pm 10.68$)$ years (range, 41-69 years). The course of disease was 2.5-24.0 years, with an average of $(13.85 \pm 4.10)$ years. The difference between the above data groups was $P>0.05$.

Inclusion criteria: Those who have been diagnosed with the disease and are in the decompensated period; The survival time was more than one year; Exclusion criteria: Lactating women; Pregnant women; Patients with heart disease; Mental illness; Those with a history of drug abuse; Thyroid disease.

\subsection{Method}

The control group was treated with conventional medicine, that is, adefovir dipivoxil capsules, oral, the initial dose of $10 \mathrm{mg}$, once a day, for 48 weeks. The observation group was treated with entecavir orally, $0.5 \mathrm{mg} /$ time, once a day for 48 weeks.

In the treatment, it is necessary to ensure the standardization of medication. In the treatment, both groups of patients need to carry out normal liver protection treatment, and carry out routine support intervention, such as ensuring the normal nutritional supply of the body. For patients with ascites, appropriate diuretics should be given, and antibiotics should be given to patients with infection. If there are adverse reactions in the treatment, it is necessary to stop medication immediately and implement targeted treatment to maintain the health of patients.

\subsection{Observation index}

Liver function indexes, liver fibrosis indexes, HBVDNA negative conversion rate and incidence of adverse reactions were compared between the two groups. Liver function index: Albumin (ALB), total bilirubin (TBIL) and alanine aminotransferase (ALT) were detected by multifunctional detector. Liver fibrosis index: Hyaluronic acid (HA), type III procollagen (III - C), type IV collagen (IV - C), laminin (LN) were collected. $6 \mathrm{ml}$ venous blood was collected, and the above indexes were detected in a multifunctional biological analyzer ${ }^{[4]}$. Adverse reactions: Nausea, vomiting, itching.

\subsection{Statistical treatment}

The measurement data (liver function index, liver fibrosis index) in this paper were represented by $(\bar{x} \pm s)$, and $\mathrm{t}$-test was performed for comparison between groups. In this paper, the counting data (HBV-DNA negative conversion rate, adverse reaction rate) were represented by $[N /(\%)]$, and the comparison between groups was tested. All data were processed with SPSS 23.0 software, and the statistical significance index was $P<0.05$.

\section{Results}

\subsection{Comparison of liver function indexes}

There was no difference in liver function between the two groups before treatment $(P>0.05)$; After treatment, the liver function indexes of the observation group were better than those of the control group, $P<0.05$, see Table 1 .

Table 1. $\mathrm{C}(\bar{x} \pm s)$

\begin{tabular}{|c|c|c|c|c|}
\hline Group & & ALB (g/L) & TBIL (umol/L) & ALT (U/L) \\
\hline \multirow[t]{4}{*}{ Observation group } & Before treatment & $30.51 \pm 5.45$ & $76.41 \pm 20.15$ & $153.57 \pm 12.66$ \\
\hline & After treatment & $37.55 \pm 6.57$ & $20.11 \pm 5.22$ & $50.60 \pm 10.31$ \\
\hline & $t$ & 5.8316 & 22.5226 & 131.2132 \\
\hline & $P$ & 0.0000 & 0.0000 & 0.0000 \\
\hline \multirow[t]{3}{*}{ Control group } & Before treatment & $30.55 \pm 5.43$ & $76.77 \pm 20.21$ & $153.44 \pm 12.70$ \\
\hline & After treatment & $32.45 \pm 6.43$ & $26.67 \pm 5.34$ & $60.70 \pm 5.47$ \\
\hline & $P$ & 0.1136 & 0.0000 & 0.0000 \\
\hline After treatment, $t$ & & 3.9228 & 6.2117 & 6.1191 \\
\hline After treatment, $P$ & & 0.0002 & 0.0000 & 0.0000 \\
\hline
\end{tabular}




\subsection{Comparison of liver fibrosis indexes}

There was no difference in liver fibrosis indexes between the two groups before treatment $(P>$
0.05). After treatment, liver fibrosis indexes in the observation group were better than those in the control group, $P<0.05$, as shown in Table 2 .

Table 2. Comparison of indicators of liver fibrosis $(\bar{x} \pm s ; n=50)$

\begin{tabular}{cccccc}
\hline Group & & HA (ug/L) & III -C (ug/L) & IV -C (ug/L) & LN (ug/L) \\
\hline Observation group & Before treatment & $245.57 \pm 50.31$ & $233.40 \pm 50.15$ & $176.61 \pm 40.35$ & $186.57 \pm 30.15$ \\
& After treatment & $105.55 \pm 20.15$ & $111.57 \pm 10.25$ & $80.15 \pm 10.54$ & $90.59 \pm 20.15$ \\
& $t$ & 18.2690 & 16.8299 & 16.3552 & 18.7152 \\
Control group & $P$ & 0.0000 & 0.0000 & 0.0000 & 0.0000 \\
& Before treatment & $244.57 \pm 50.25$ & $232.20 \pm 50.13$ & $176.55 \pm 40.22$ & $186.55 \pm 30.20$ \\
& After treatment & $115.57 \pm 20.20$ & $126.57 \pm 10.35$ & $90.15 \pm 10.68$ & $100.57 \pm 20.20$ \\
After treatment, $t$ & $t$ & 2.4833 & 14.5918 & 4.7124 & 16.7333 \\
After treatment, $P$ & $P$ & 0.0147 & 0.0000 & 0.0000 & 0.0000 \\
\hline
\end{tabular}

\subsection{Comparison of HBV-DNA negative conversion} rate

Observation group: The negative rate of HBV-DNA was $92.0 \%$; In the control group: The negative rate of HBV-DNA was $76.0 \%(38 / 38), P<0.05$.

\subsection{Incidence of adverse reactions}

Observation group: There were 1 cases of nausea, 1 case of vomiting and 1 case of pruritus. The incidence of adverse reactions was $6 \%$. In the control group: There were 2 cases of nausea, 1 case of vomiting and 2 cases of pruritus, and the incidence rate was $10 \%$. There was no statistical difference between the two groups $(P<0.05)$.

\section{Discussion}

Chronic hepatitis B is a frequently occurring disease, which is due to the replication and function of the virus in the body of patients, and the released antigen causes liver inflammation, leading to liver fibrosis, and eventually leading to liver cirrhosis ${ }^{[5]}$. There are many causes of liver cirrhosis, most of which are caused by hepatitis virus infection. When the body is infected with hepatitis B virus, the virus will enter the liver nucleus, and then the virus will continue to replicate, damaging the liver nucleus of patients ${ }^{[6]}$. In this process, the virus continuously segmented the liver, resulting in regenerative nodules in the fibrous tissue of the liver, thus forming more fibrous tissue, increasing the hardness of the liver and causing cirrhosis $^{[7]}$. When patients with cirrhosis, because the liver does not have normal liver function, it is easy to increase the pressure of portal vein, which makes the blood flow back and cause liver bleeding.
The occurrence of chronic hepatitis B cirrhosis has a certain period of time, if not treated in time, it will increase the complications. With the progress of the disease, when the patients enter the stage of decompensation, the disease is in the late stage, so it is necessary to carry out antiviral treatment in time to control the disease. At present, entecavir has been used in the treatment of decompensated chronic hepatitis B cirrhosis, and has achieved certain therapeutic effect. Entecavir is a new type of nucleotide, which has the effect of anti-hepatitis virus, and the drug resistance rate is low. After taking entecavir, patients have better tolerance, and there will be no serious adverse reactions after medication, so it has high safety ${ }^{[8]}$. In addition, entecavir can inhibit the activity of hepatitis B virus, block the recovery ability of hepatitis $\mathrm{B}$ virus, and improve liver function ${ }^{[9]}$.

In conclusion, entecavir is effective in the treatment of patients with decompensated chronic hepatitis B cirrhosis, which is worthy of promotion.

\section{References}

[1] Xue LN, Zhu GB. The effect of entecavir dispersible tablets on patients with decompensated hepatitis B cirrhosis [J]. Systematic medicine, 2020, 5 (20): 50-52.

[2] Wang H. Clinical efficacy of entecavir in the treatment of decompensated hepatitis B cirrhosis patients [J]. Chinese medical guide, 2020,18 (27): 35-36.

[3] Sun PI, Tao GY, Zheng HK. Efficacy and safety of entecavir in patients with decompensated hepatitis B cirrhosis[J]. Shenzhen Journal of integrated traditional Chinese and Western medicine, 2020, 30 (15): 149-150

[4] Zhang LL, Liu T, Ma H, et al. Entecavir in the treatment of 
decompensated hepatitis B cirrhosis [J]. Western medicine, 2020, 32 (7): 999-1002+ 1006

[5] Tang ZZ, Chen HY, Lin WB. Effect of entecavir on liver function and complications in patients with decompensated hepatitis B cirrhosis [J]. Capital food and medicine, 2020, 27 (13): 90.

[6] Wang YQ, Chen CJ. Effect of adefovir dipivoxil combined with entecavir on serum cytokines in elderly patients with decompensated hepatitis B related cirrhosis [J]. Chinese University Medical Journal, 2020, 34 (5): 385-386 + 390 .
[7] Sun HY, Liu GQ. Effect of entecavir on patients with decompensated chronic hepatitis B cirrhosis [J]. Chinese Journal of practical medicine, 2020, 15 (4): 100-101.

[8] Yuan WH. Short term efficacy and safety analysis of entecavir in the treatment of patients with decompensated chronic hepatitis B cirrhosis [J]. Electronic Journal of general Stomatology, 2019, 6 (35): 186-190.

[9] Cai LN, Yuan YB. Efficacy and safety of entecavir in the treatment of decompensated chronic hepatitis B cirrhosis [J]. Modern diagnosis and treatment, 2019, 30 (2): 250-252. 